\title{
Standardising the organisation of clinical equipment on surgical wards at North Bristol NHS Trust: a quality improvement initiative
}

Joseph Ward, Robin Spencer, Eleanor Soo, katherine finucane

North Bristol NHS Trust

\begin{abstract}
Poorly organised clinical equipment can waste significant amounts of time otherwise available for direct patient care. As a group of foundation year one doctors, we identified the organisation of clinical equipment across surgical wards at North Bristol NHS Trust to be poor with stocks often low and items frequently difficult to locate. Time-motion studies $(n=80)$ were confirmatory demonstrating that the mean time to collect equipment necessary for venepuncture, cannulation, arterial blood gases, or blood cultures ranged from 121 to 174 seconds between different areas.

By applying a plan-do-study-act (PDSA) methodology, surveying peers as well as working with nursing staff and senior managers, we were able to purchase and implement clinical equipment trolleys on 10 surgical wards across the trust to reduce the time-taken to locate clinical equipment to between 38 to 45 seconds $(p=0.01)$. We feel the key factors for the success of our initiative were strong multidisciplinary engagement and a simple uniform idea. Clinical equipment trolleys organised in a standardised manner have now been rolled out hospitalwide in the new Southmead Hospital Brunel building.
\end{abstract}

\section{Problem}

Across the surgical wards at North Bristol NHS Trust, a large degree of variation existed in the organisation, location and availability of disposable clinical equipment. Junior doctors, nurses, and ward staff commonly wasted significant periods of time trying to locate clinical equipment such as cannulas, blood sample bottles, and syringes that were poorly labelled, difficult to find or sometimes simply absent. This frequently resulted in significant frustration for clinical staff and time wasted that could otherwise be better spent on providing direct patient care.

\section{Background}

In similarity with many hospitals, the surgical wards at North Bristol NHS Trust are busy clinical areas that see high numbers of elective and emergency admissions. Each ward has resident nursing staff but doctors and allied health professionals tend to work across wards caring for patients and may be required to work hospital-wide whilst on-call. Due to the nomadic-nature of working practices accompanied by frequent short four month rotations, junior doctors in particular felt unfamiliar with the organisation and availability of clinical equipment across wards having sometimes only rarely visited particular clinical areas.

On each ward, clinical equipment was organised by a designated member of the nursing staff responsible for its ordering, restocking and lay out. Due to difficulties with storage, a high workload, and sporadic ordering practices, clinical equipment rooms were frequently overstocked with rarely used items and understocked with commonly used items. This led to situations where junior doctors requiring a specific piece of clinical equipment, eg arterial blood gas syringe or blood culture bottles had to leave a ward to locate items. More frustratingly, it was common for junior doctors to be unable to locate equipment even on well-stocked and organised wards because they were simply unaware where the equipment was located.

To complicate matters further, clinical equipment rooms were frequently overcrowded spaces located away from areas of direct patient care due to historically cramped wards. From a financial perspective, junior doctors were also unaware of the price of individual disposable items meaning that some equipment was used wastefully (see figure 1: Heterogeneity of clinical equipment room arrangement, layout and organisation).

Organisation of clinical equipment is a key tenet of the NHS Institute for Innovation's "Productive Ward" strategy.[1] The strategy aims to increase time available to clinicians for direct patient care by improving ward environments and processes to enhance safety, efficiency and cost-effectiveness. At the time of the quality initiative, this strategy was being rolled out across surgical wards at North Bristol by senior nurses keen to work with junior doctors. Concurrently, NHS Education South West (the body responsible for training doctors locally) was keen to promote the involvement of junior doctors in quality improvement and encourage the development of management and leadership skills by running quality improvement workshops led by local consultants.

As a group of keen and motivated foundation year one doctors, we felt passionately that the organisation of clinical equipment on surgical wards at North Bristol could be improve to enhance our working lives, save the trust money and increase the time available to provide hands-on clinical care. 


\section{Baseline measurement}

As a group of eight junior doctors led by a consultant, we performed a baseline evaluation of the problem using time-motion studies. This involved measuring the time taken by each team member to locate specific pieces of clinical equipment necessary for venepuncture, cannulation, arterial blood gas measurement, and blood cultures on unfamiliar wards. Having performed 80 experiments, we found that the mean time to collect equipment for each of the four procedures ranged from 121 to 174 seconds across ten wards (see figure 2) with the times taken to locate venepuncture, cannulation, arterial blood gas and blood cultures: 133 secs, 124 secs, 121 secs, and 174 secs respectively (Figure 2: Powerpoint Slide 2 - Baseline Measure (PDSA 1) - Seconds taken to locate clinical equipment: how much time is wasted?).

See supplementary file: ds5129.pptx - "Figure 1-6"

\section{Design}

Having identified an important clinical problem, we brainstormed potential solutions as a group. These included re-designing clinical equipment rooms, rolling out clinical equipment trolleys, or establishing a central clinical equipment repository.

We were particularly keen to understand experiences of colleagues elsewhere and were made aware of a solution called the DocBox (see figure 3). The concept had been rolled out across six surgical wards at University Hospitals Bristol NHS Foundation Trust with clinical equipment organised into a standardised layout of bright yellow wall-mounted trays. Each DocBox was located in a prominent position inside each clinical equipment area and accompanied by an organisational plan. The initiative had proven very successful with measured reductions in the time spent by oncall doctors locating clinical equipment and a positive reception from ward-staff.

Considering the initiative, we decided against trying to implement a similar concept locally because it would have required significant alteration to the fittings and layout of busy clinical areas and necessitated permissions, significant costs and disruption to patient care. In addition, some wards had enough space for the DocBox whilst others simply did not. Considering the other options, we felt a central repository was unworkable but that clinical equipment trolleys would be a more versatile, compact solution negating the need for alterations whilst being easily recognisable and cheaper (at £270 per trolley). We also felt trolleys could be placed close to patients and located in positions that would most useful for wardstaff.

Having decided to pursue the trolley concept, we were keen that they should become an integrated part of the ward furniture and resident staff should feel ownership of their trolley ensuring maximum use. We resolved at an early stage to engage nursing colleagues by discussing the trolleys informally, considering with each ward where a trolley might go and asking for suggestions, comments, and help with their implementation.

\section{Strategy}

Having consulted widely to reach a consensus that clinical equipment trolleys were the best local fit, we contacted senior trust managers with the aim of purchasing a single clinical equipment trolley. After much e-mail communication, we secured funding for a single pilot clinical equipment trolley for roll out on a short-stay ward.

Implementation of the first trolley was pivotal for the success of the initiative allowing us to conduct several plan-do-study-act (PDSA) data collection cycles and confirm that the trolleys were effective at reducing time taken to locate equipment. The PDSA cycles and their results are summarised in figure 4 but allowed us to refine and optimise trolley organisation. Collected data allowed us to ensure that the most frequently used pieces of clinical equipment were located in the highest drawers of each trolley to be most accessible, whilst less commonly used equipment was clearly labelled but lower down.

Concurrently to the implementation of our first clinical equipment trolley, we undertook a short electronic questionnaire of junior doctors to gauge how we could make the trolleys as useful, welldesigned and time-saving as possible. The questionnaire surveyed respondents on equipment that was most frequently required (and should be stocked) as well as the best ways to organise the trolleys. Less formally, we also contacted senior nursing staff on nominated wards to ask if they would be happy for a clinical equipment trolley to be located within their clinical areas.

Finally armed with the results of a supportive e-survey, evidence of clear time-savings, a list of receptive host wards, estimated costs and clear roll-out plan, we sought a meeting with trust's director of operations. At this meeting we outlined our initiative, presented evidence for its effectiveness as well as a plan for further implementation. A successful pitch allowed us to secure ring-fenced funding to roll out trolleys on ten surgical wards across the trust collecting PDSA data pre- and post-implementation.

\section{Results}

Results of junior doctor survey: sixty-one responses (24 Foundation Year 1 doctors, 24 senior house offices, and 13 specialist registrars) were received from junior doctors across North Bristol. $96.7 \%$ of respondents agreed with the statement "an easily identifiable clinical equipment trolley organised in a standardised manner and present on every ward at NBT would be useful." Respondents were asked to estimate the estimated time-saving that a clinical equipment trolley would provide to them during the course of a normal working day and during an on-call shift (see tables 1 and 2).

Results of PDSA data collection: five PDSA data collection cycles were undertaken during the course of the initiative with the cycle findings and changes summarised in table 3 . Over the course of the initiative the time taken to locate clinical equipment reduced for all four clinical tasks from 121 to 174 seconds to 38 to 45 seconds 
$(p=0.01)$ and is further summarised in figure 4.

See supplementary file: ds5156.docx - "Tables 1-3"

\section{Lessons and limitations}

During the course of our quality improvement initiative, we learned the following lessons:

\section{Multidisciplinary engagement and ownership:}

multidisciplinary engagement was a key aspect of our initiative's success particularly during the roll out stages when interdisciplinary team-work was vital. As rotating junior doctors, it was important to us that the trolleys were fully utilised once we had moved on so we engaged with ward staff to ensure the clinical equipment trolleys were appropriate for each ward, optimally located, and organised in a way that would aid direct patient care so that ward took ownership of their trolley and brought into the concept.

2. Uniformity: this was important for ensuring that our measured time-savings were maintained when the trolleys were rolled out across multiple wards allowing medical staff to be familiar with the trolleys no matter which ward they were visiting. We feel uniformity of design has a strong role to play across the NHS improving the safety of processes and enhancing efficiency.

3. Co-ordination and leadership: from the outset of the initiative, one person (RS) was designated responsible for co-ordinating the project, arranging meetings, and liaising with senior colleagues. This meant that despite being a large group of junior doctors with time-consuming clinical duties and varying interests, the project was well led and progressed contemporaneously.

4. Importance of data: early on in our project, the validity of our idea was supported by clear data demonstrating the proof of concept. This allowed us to confidently advocate the effectiveness of our initiative to trust managers and provided the evidence we needed to strengthen our case when requesting funding. As we progressed, the more data we collected, the stronger our case became.

5. A can-do attitude: We came across a number of obstacles during the course of our project that at times seemed daunting. This was particularly true when procuring the trolleys through NHS supply chain and when storing and prepping the trolleys pre-roll out. We certainly found that perserverance and a positive attitude paid off in buckets here.

6. Listening to feedback (good and bad): During the course of the project, we had positive and negative feedback either objectively driven by the PDSA data or subjectively given at meetings. All feedback was helpful in allowing us to improve the trolleys, for instance prompting us to place item pictures and cost labels within each drawer and more appropriately position the trolleys.

Limitations

1. Sidelining of trolleys: We tried to locate clinical equipment trolleys in locations that were as close to patient care as possible but did not obstruct the coming and going of wardstaff and visitors. We found that trolleys on some wards due to their mobile nature were sometimes sidelined to out-of the way locations whilst on other wards they were simply placed in already crowded clinical equipment rooms but we tried to discourage this.

2. Effecting hospital wide change: Initially when we set out to pursue our quality improvement initiative, we envisaged rolling out the concept to all wards across the trust. It quickly became apparent that this aim would be undeliverable over the two year time-frame of our foundation training so we took the decision to restrict the initiative to the more achievable remit of the surgical directorate. Nonetheless, now that many of us have all moved on beyond foundation training, the project has been taken forward by the local foundation programme lead and trolleys have been incorporated into the design of every ward in the new Southmead Hospital Brunel Building (See figure 6).

\section{Conclusion}

By implementing clinical equipment trolleys on the surgical wards at North Bristol NHS Trust, we were able to improve the accessibility and availability of clinical equipment to all healthcare professionals within these clinical areas. By implementing, a very simple commonsense intervention with multidisciplinary engagement, supportive managers, and a versatile adaptive strategy we achieved a measured reduction in the time-taken to locate clinical equipment. Standardising the organisation of clinical equipment across clinical areas and even across trusts has the potential to considerably reduce clinical time wasted looking for clinical equipment and increase time available for medical staff to provide direct patient care.

\section{References}

1. NHS Institute for Improvement and Innovation: Releasing Time to Care

www.institute.nhs.uk/quality_and_value/productivity_series/producti ve ward.html

Last accessed: 29th October 2014.

1. Bethune R, Soo E, Woodhead P, Van Hamel C, Watson J. Engaging all doctors in continuous quality improvement: a structured, supported programme for first-year doctors across a training deanery in England. BMJ Qual Saf 2013;22(8)613-7.

\section{Declaration of interests}

JW, RS, EN-S, and KF have no competing interests to declare. 


\section{BMJ Quality Improvement Reports}

We acknowledge the contributions of Jane Muir, Rachel Cowie, Phil Williams, Natalie Parkinson, Yusra Khan, Hajib Kamali and Ben Walton as part of the original QI team. We also acknowledge the support and funding provided by North Bristol NHS Trust postgraduate medical education department, surgical directorate and executive team as well as NHS Medical Education South West who facilitated and funded our initiative. We thank Matt Molyneux, Ed Miles, and the DocBox team at University Hospitals Bristol NHS Foundation Trust for sharing their approach to a very similar problem with us.

\section{Ethical approval}

Ethical approval was not sought for this project. 